American Journal of Applied Sciences 7 (8): 1038-1042, 2010

ISSN 1546-9239

(C) 2010 Science Publications

\title{
Eco-Friendly Synthesis of Fucoidan-Stabilized Gold Nanoparticles
}

\author{
${ }^{1}$ Suwicha Soisuwan, ${ }^{2}$ Warangkana Warisnoicharoen, \\ ${ }^{3}$ Kriengsak Lirdprapamongkol and ${ }^{3}$ Jisnuson Svasti \\ ${ }^{1}$ Pharmaceutical Technology (International Program), \\ ${ }^{2}$ Department of Food and Pharmaceutical Chemistry, \\ Faculty of Pharmaceutical Sciences, \\ Chulalongkorn University, Bangkok 10330, Thailand \\ ${ }^{3}$ Laboratory of Biochemistry, Chulabhorn Research Institute, \\ Vibhavadee-Rangsit Highway, Laksi, Bangkok 10210, Thailand
}

\begin{abstract}
Problem statement: Metallic gold nanoparticles (AuNPs) are widely used in many applications including medical, pharmaceutical, diagnostics and sensors. The chemical synthesis of AuNPs normally requires synthetic materials which might cause the toxicological concerns. The use of naturally occurring materials like fucoidans for successful synthesis of AuNPs is of interests. Approach: Fucoidans as sulfated polysaccharides from marine algae Cladosiphon okamuranus (ofucoidan) and Kjellamaniella crassifolia (t-fucoidan) were used for synthesis of AuNPs. The suitable condition for the synthesis was investigated to obtain the nanometric size of AuNPs. The synthesized AuNPs were characterized for the size, morphology and Surface Plasmon Resonance (SPR). Results: The fucoidan-stabilized AuNPs containing optimum weight ratio of gold atom to fucoidan yielded the spherical sizes with an average of 8-10 $\mathrm{nm}$ and the absorption maxima of SPR band around 527-530 $\mathrm{nm}$. The AuNPs stabilized by o-fucoidan structured as linear polymer were more monodisperse than those stabilized by t-fucoidan structured as branched polymer. Conclusion: Fucoidan can be employed solely for AuNP synthesis and the sulfate constituent in fucoidan is important for gold reduction and stabilization. The simple yet eco-friendly synthesis of AuNPs stabilized by fucoidans would be attractive for application use of metallic nanoparticles.
\end{abstract}

Key words: Gold nanoparticles, fucoidan, polysaccharides, synthesis

\section{INTRODUCTION}

Gold nanoparticles (AuNPs) are attractive for many applications including pharmaceuticals, diagnostics and biosensors (Ghosha et al., 2008; Zhao et al., 2008; Peng et al., 2009). The advantages of AuNPs come from their biocompatibility and ability to target delivery of therapeutic agent (Patra et al., 2008). The synthesis of AuNPs normally involves a reduction of positively charged gold atom and an addition of stabilizing agents. The chemical used for gold synthesis is normally citrate, sodium borohydride and organic compounds (Sun et al., 2003; Kimling et al., 2006). In order to reduce the use of chemical and toxic waste to the environment, the synthesis of AuNPs using natural compounds is of interests. Among them, polysaccharide or polysaccharide-based biopolymer has been employed to synthesize AuNPs. Polysaccharides that have been recently reported for synthesis of AuNPs include all those containing amine groups in particular chitosan, heparin and hyaluronan (Huang and Yang, 2004a; 2004b; Kemp et al., 2009). In addition, the ability of dried seaweed Sargasssum wightii to stabilize AuNPs was thought to arise from extracellular polysaccharides present in seaweed (Singaravelu et al., 2007).

Fucoidan is a polysaccharide extract from cell wall of marine brown algae such as Laminariales, Fucales, Chordariales, Desmarestiales and Dictyotales. Fucoidan shows some pharmacological activities including anti-inflammation, anti-coagulant, anti-viral, anti-cancer and anti-peptic ulcer activities (Shibata et al., 2003; Yang et al., 2006; Cumashi et al., 2007; Teruya et al., 2007; Li et al., 2008). Moreover, fucoidans are also applied in cosmetic application as anti-aging compound and whitening agent (Fitton et al., 2007). The fucoidan

Corresponding Author: Warangkana Warisnoicharoen, Department of Food and Pharmaceutical Chemistry, Faculty of Pharmaceutical Sciences, Chulalongkorn University, Bangkok 10330, Thailand Tel: + 6622188332 Fax: +66 22188332 
structure is a linear backbone of $\alpha-1 \rightarrow 3$-linked fucopyranose with additional sulfate group and may have a branched structure (Li et al., 2008). Nonetheless, there is no evidence whether fucoidan can facilitate the formation of AuNPs. The green synthesis of AuNPs using natural fucoidans would provide an alternative to chemical method. The aim of study is to synthesize AuNPs using fucoidan extract from marine algae and the characteristics of synthesized AuNPs are investigated.

\section{MATERIALS AND METHODS}

The two different species of marine algae, Okinawa mozuku fucoidan from Cladosiphon okamuranus (o-fucoidan; $\mathrm{M}_{\mathrm{w}}=380,000 \mathrm{~g} \mathrm{~mol}^{-1}$ ) and Takara kombu fucoidan from Kjellamaniella crassifolia (t-fucoidan; $\mathrm{M}_{\mathrm{w}}=200,000 \mathrm{~g} \mathrm{~mol}^{-1}$ ) were obtained from Toyo Science and Takara Bio, Japan. The structures of o-fucoidan and t-fucoidan are represented in Fig. 1. Hydrogen tetrachloroaurate (III) trihydrate $\left(\mathrm{HAuCl}_{4} \cdot 3 \mathrm{H}_{2} \mathrm{O}\right)$ solution $(30 \% \mathrm{w} / \mathrm{w})$ was purchased from Sigma (St. Louis, USA). All chemicals were used as received.

Synthesis and characterization of gold nanoparticles: Fucoidan was dissolved in $50 \mathrm{~mL}$ deionized water to obtain various concentrations $(0.01$, $0.02,0.04,0.06,0.08,0.10,0.12,0.14,0.16,0.20$ and $0.40 \% \mathrm{w} / \mathrm{v})$. Then, $3.45 \mu \mathrm{L}$ of $30 \% \mathrm{w} / \mathrm{w}$ hydrogen tetrachloroaurate (III) trihydrate $\left(\mathrm{HAuCl}_{4} \cdot 3 \mathrm{H}_{2} \mathrm{O}\right)$ solution was added to obtain $20 \mathrm{ppm}$ of gold atom. The mixture was heated to $80^{\circ} \mathrm{C}$ on a water bath with magnetic stirring for $45 \mathrm{~min}$. The mixture was visually observed for appearance and determined for Surface Plasmon Resonance (SPR) spectrophotometrically using Shimadzu UV-Vis 160A. The morphology and size of AuNPs were visualized using Transmission Electron Spectroscopy (TEM) on a 200 KV Joel JEM2100 equipped with Semafore 4.01 program for size analysis and the zeta potential was determined using Zetasizer NanoZS( ZEN3600).

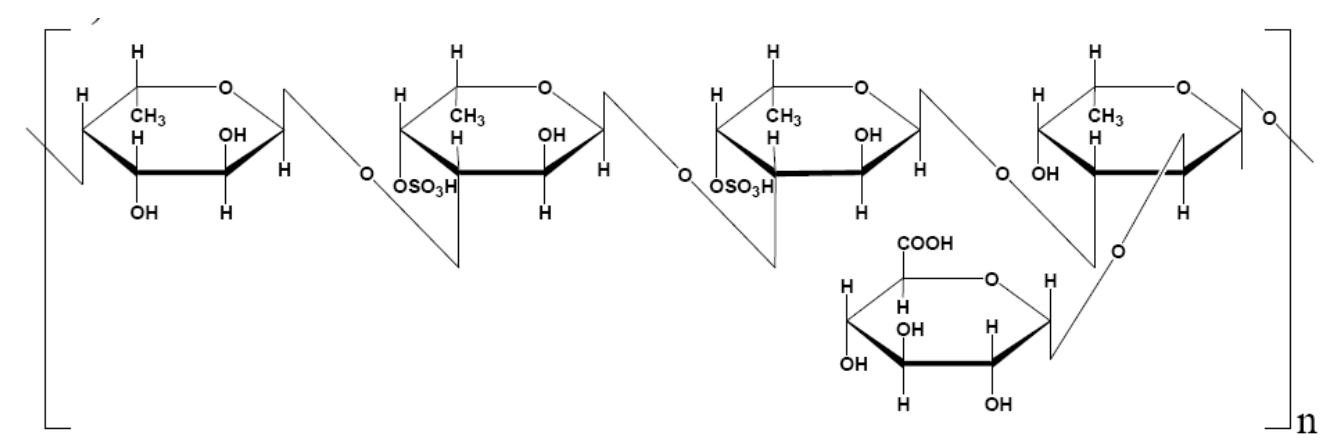

(a)

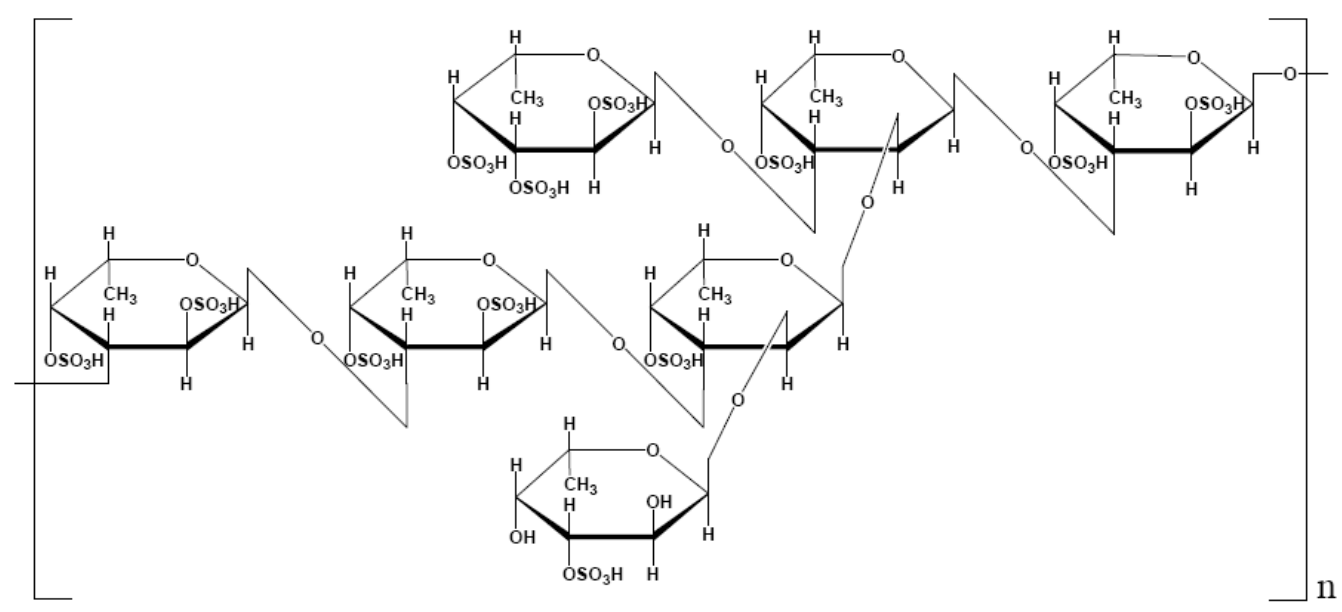

(b)

Fig. 1: Structures of o-fucoidan (a) and t-fucoidan (b) 
For the study on the effect of duration time on the synthesis of fucoidan-stabilized AuNPs at ambient temperature, the fucoidan was weighed and dissolved in water to obtain $0.04 \% \mathrm{w} / \mathrm{v}$ for o-fucoidan and $0.10 \% \mathrm{w} / \mathrm{v}$ for $\mathrm{t}$-fucoidan before the gold solution was added. The mixture was stirred until the ruby-red color of AuNP solution appeared. The synthesized AuNPs were determined for the change in SPR band at $1,8,24,48$ and $72 \mathrm{~h}$.

\section{RESULTS}

From the result, red solution of AuNPs was observed when fucoidan concentrations were in the range of $0.01-0.12 \% \mathrm{w} / \mathrm{v}$ (Fig. 2) which corresponded to the weight ratios of gold atom to fucoidan ranging from 1:50 to 1:60. The SPR bands of AuNPs containing gold to fucoidan at different weight ratios, $1: 5,1: 10,1: 20,1: 30,1: 40,1: 50$ and 1:60, were further characterized. The overall peak absorbance of the nanoparticles was downward to blue shift for both fucoidans upon decreasing weight ratio of gold to fucoidan, from $530 \mathrm{~nm}$ (1:5 ratio) to $519 \mathrm{~nm}(1: 60$ ratio) for o-fucoidan (Fig. 3a), and from $547 \mathrm{~nm}$ (1:5 ratio) to $522 \mathrm{~nm}$ (1:60 ratio) for t-fucoidan (Fig. 3b). The weight ratios of gold atom to fucoidan which yielded the absorption maxima, $\mathrm{A}_{\max }$, were 1:20 $\left(\mathrm{A}_{\max }=\right.$ $0.39,527 \mathrm{~nm})$ for o-fucoidan and 1:50 $\left(\mathrm{A}_{\max }=0.38\right.$, $529.5 \mathrm{~nm})$ for $\mathrm{t}$-fucoidan. Upon decreasing the ratio beyond their optimum points, lower absorbance of ofucoidan stabilized AuNPs (o-AuNPs) and AuNPs stabilized by $\mathrm{t}$-fucoidan (t-AuNPs) was found. Although the SPR bands of AuNPs stabilized by either o-fucoidan or $\mathrm{t}$-fucoidan were similar, we observed that the appearance of o-AuNPs was ruby red while those stabilized by $\mathrm{t}$-AuNPs was red purple. The TEM images showed the morphology of spherical o-AuNPs and tAuNPs at 1:20 and 1:50 weight ratios of gold to ofucoidan and t-fucoidan, respectively (Fig. 4). The TEM results indicated that the average size of o-AuNPs $(8.54 \pm 2.96 \mathrm{~nm}, \mathrm{n} \geq 400)$ became smaller and narrower in size distribution than $\mathrm{t}-\mathrm{AuNPs}(10.74 \pm 4.78 \mathrm{~nm}, \mathrm{n} \geq 400)$.

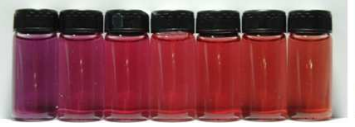

(a)

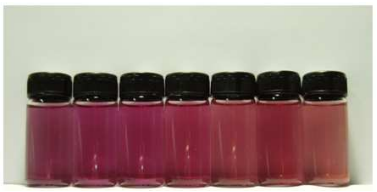

(b)
Fig. 2: Appearance of AuNPs stabilized by o-fucoidan (a) and t-fucoidan (b) upon decreasing weight ratios of gold atom to fucoidan (from left to right), 1:5, 1:10, 1:20, 1:30, 1:40, 1:50 and 1:60
Since the fucoidan contains chemical constituents with negative charges such as sulfate, the zeta potential of AuNPs were expectedly in negative values, $-34.70 \pm 4.99 \mathrm{mV}(\mathrm{n}=4)$ for o-AuNPs and $-28.50 \pm 3.66$ $\mathrm{mV}(\mathrm{n}=4)$ for $\mathrm{t}$-AuNPs. In another experiment, we demonstrated if fucoidan-stabilized AuNPs can be simply synthesized without the need of heat. The weight ratios of gold to fucoidan for synthesis were 1:20 and 1:50 for o-AuNPs and t-AuNPs, respectively. The sample was rigorously stirred at room temperature for different time periods and the SPR band of AuNPs was observed. The results showed the effect of time period on the peak absorbance of AuNPs. The highest absorbance of o-AuNPs and t-AuNPs was obtained within $48 \mathrm{~h}$ (Fig. 5).

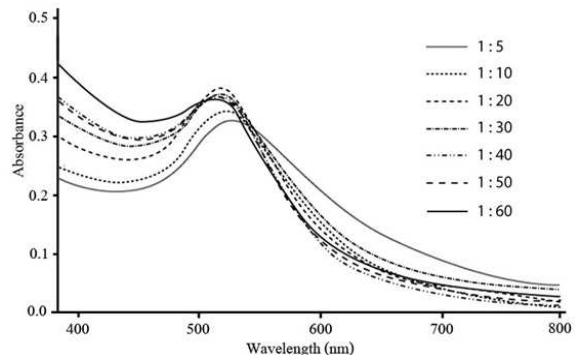

(a)

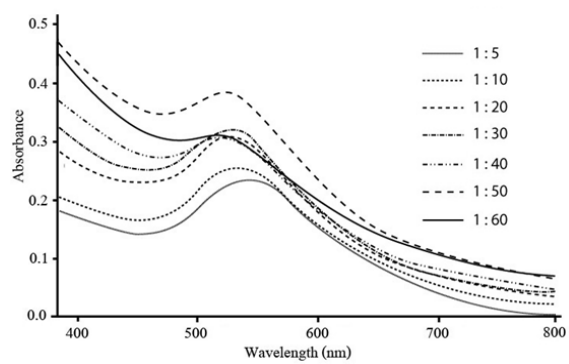

(b)

Fig. 3:UV-Vis spectrum of fucoidan-stabilized AuNPs prepared at $80^{\circ} \mathrm{C}$ using various weight ratios of gold to o-fucoidan (a) and t-fucoidan (b)

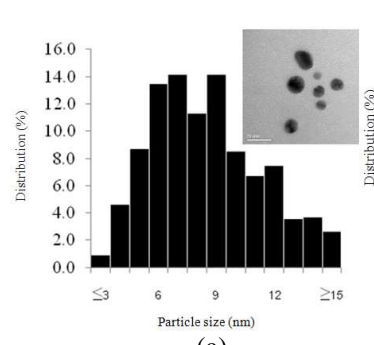

(a)

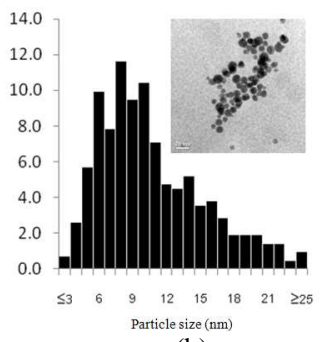

(b)
Fig. 4:TEM images (inset, scale bar of $20 \mathrm{~nm}$ ) and histogram of size distribution of o-fucoidanstabilized AuNPs (a) and t-fucoidan-stabilized AuNPs (b) 


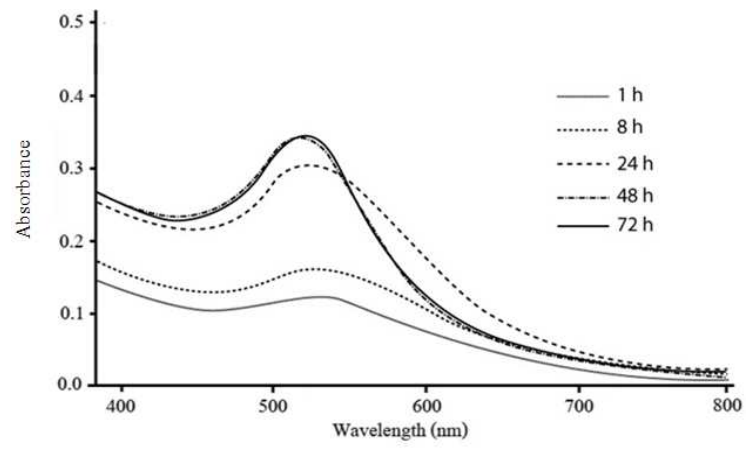

(a)

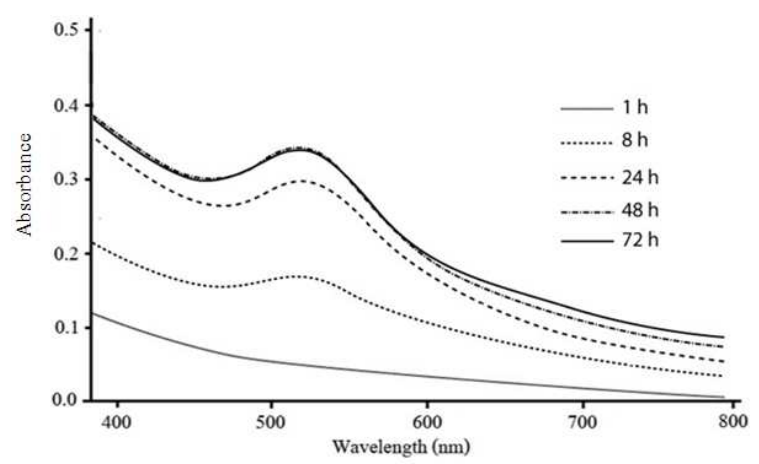

(b)

Fig. 5:UV-Vis spectrum of fucoidan-stabilized AuNPs prepared at ambient temperature and different time, using weight ratios of 1:20 gold to ofucoidan (a) and 1:50 gold to t-fucoidan (b)

\section{DISCUSSION}

The ruby-red color of the gold solution usually indicates the presence of AuNPs in the size range below $25 \mathrm{~nm}$, which corresponds to the SPR band around 520$530 \mathrm{~nm}$ (Link and El-Sayed, 1999). The red-purple color of t-AuNPs seemed to correlate with the red shift of the absorbance peak to longer wavelengths than those of o-AuNPs, compared at the same ratio. The red shift at absorption maxima may also indicate the aggregation behavior of nanoparticles (Lee and PerezLuna, 2005; Schofield et al., 2007). Normally, the AuNPs having size of less than $25 \mathrm{~nm}$ show the SPR band of lower than $530 \mathrm{~nm}$ or so-called the intrinsic size region where the wavelength shift in the absorption maxima is not significant and the measured absorbance directly determines the concentration of nanoparticles present (Link and El-Sayed, 1999). AuNPs at the absorption maxima were consequently used for further studies on size distribution and homogeneity of nanoparticles by using TEM analysis. Although the fucoidans from two different species of marine algae can facilitate the formation of AuNPs within the 10-nm size range, we observed some differences in that $\mathrm{t}-$
AuNPs were less monodispersed and more aggregated in nature. The findings from TEM seemed to support our SPR results earlier on the longer wavelength at absorption maxima of $\mathrm{t}$-AuNPs.

Although o-fucoidan and t-fucoidan initiated the formation of nanometer-sized AuNPs, the difference in SPR spectrum and size distribution noticed might be arisen from fucoidan structure. While polymer chain of o-fucoidan composes of mainly linear chain of $\alpha$ $(1 \rightarrow 3)$-linked sulfated fucopyranose with substituting residues of glucuronic acid at the $\mathrm{C}-2$ position of $\mathrm{L}$ fucopyranose for every 4 molecules of the main chain. In contrary, a branched structure of sulfated fucopyranose was found in t-fucoidan at C-2 position of the main chain. Since sulfate group is the site for gold reduction and stabilization, amount and location of the chemical group are likely to be responsible for nanoparticle dispersity. For t-fucoidan, the sulfate esters present in a main chain together with additional ones at side chains can possibly interact with several nanoparticles, hence raising them to stay in contact with each other and cause the occurrence of gold nanocluster. Whether the other factors namely molecular weight and concentration of o-fucoidan and t-fucoidan may affect the AuNP dispersity are questionable. However, from the study of Huang and Yang (2004a) on chitosan-stabilized AuNPs, the fivefold lower in concentration and eight-fold less in molecular weight of chitosan did not obviously cause the nanoparticles more dispersity. In addition, oAuNPs and t-AuNPs were able to be synthesized at room temperature and the peak absorbance was in the time-dependent manner up to $48 \mathrm{~h}$. The findings were in agreement with other report on the time duration required for complete formation of AuNPs synthesized using dried seaweed at an ambient temperature (Singaravelu et al., 2007).

\section{CONCLUSION}

Conclusively, the present study provides an alternative for eco-friendly synthesis of spherical and monodisperse AuNPs using sulfated polysaccharides from algae fucoidans. The different structure of fucoidan is less likely to significantly impact on nanoparticle formation but does affect on the SPR, size distribution and dispersity. Fucoidan-stabilized metallic AuNPs can be further investigated for their potential in various nanotechnology-based applications.

\section{ACKNOWLEDGEMENT}

The researchers want to thank Dr. Hideo Saito, Toyo Science and Takara Bio Japan, for fucoidan used in the study. The partially financial support from the 
Faculty of Graduate Studies, Chulalongkorn University is acknowledged.

\section{REFERENCES}

Cumashi, A., N.A. Ushakova, M.E. Preobrazhenskaya, A. D'Incecco and A. Piccoli et al., 2007. A comparative study of the anti-inflammatory, anticoagulant, antiangiogenic and antiadhesive activities of nine different fucoidans from brown seaweeds. Glycobiology, 17: 541-552. DOI: 10.1093/glycob/cwm014

Fitton, J.H., M. Irhimeh and N. Falk, 2007. Macroalgal fucoidan extracts: A new opportunity for marine cosmetics. Cosmet. Toiletr., 122: 55-64. http://www.eis.hu.edu.jo/Deanshipfiles/pub10697657.pdf

Ghosha, P., G. Hana, M. Dea, C.K. Kima and V.M. Rotello, 2008. Gold nanoparticles in delivery applications. Adv. Drug Del. Rev., 60: 1307-1315. DOI: 10.1016/j.addr.2008.03.016

Huang, H. and X. Yang, 2004a. Synthesis of chitosanstabilized gold nanoparticles in the absence/presence of tripolyphosphate. Biomacromolecules, 5: 2340-2346. DOI: 10.1021/bm0497116

Huang, H. and X. Yang, 2004b. Synthesis of polysaccharide-stabilized gold and silver nanoparticles: A green method. Carbohyd. Res., 339: 2627-2631. DOI: 10.1016/j.carres.2004.08.005

Kemp, M.M., A. Kumar, S. Mousa, T.J. Park and P. Ajayan et al., 2009. Synthesis of gold and silver nanoparticles stabilized with glycosaminoglycans having distinctive biological activities. Biomacromolecules, 10: 589-595. DOI: 10.1021/bm801266t

Kimling, J., M. Maier, B. Okenve, V. Kotaidis and H. Ballot et al., 2006. Turkevich method for gold nanoparticle synthesis revisited. J. Phys. Chem. B., 110: 15700-15707. DOI: 10.1021/jp061667w

Lee, S. and V.H. Perez-Luna, 2005. Dextran-gold nanoparticle hybrid material for biomolecule immobilization and detection. Anal. Chem., 77: 7204-7211. DOI: 10.1021/ac050484n

Li, B., F. Lu, X. Wei and R. Zhao, 2008. Fucoidan: Structure and bioactivity. Molecules, 13: 1671-1695. DOI: $10.3390 /$ molecules 13081671

Link, S. and M.A. El-Sayed, 1999. Spectral properties and relaxation dynamics of surface Plasmon electronic oscillations in gold and silver nanodots and nanorods. J. Phys. Chem. B., 103: 8410-8426. DOI: $10.1021 /$ jp9917648
Patra, C.R., R. Bhattacharya, E. Wang, A. Katarya and J.S. Lau et al., 2008. Targeted delivery of gemcitabine to pancreatic adenocarcinoma using cetuximab as a targeting agent. Cancer Res., 68: 1970-1978. DOI: 10.1158/0008-5472.CAN-076102

Peng, G., U. Tisch, O. Adams, M. Hakim and N. Shehada et al., 2009. Diagnosing lung cancer in exhaled breath using gold nanoparticles. Nat. Nanotechnol., 4: 669-673. DOI: 10.1038/nnano.2009.235

Schofield, C.L., R.A. Field and D.A. Russell, 2007. Glyconanoparticles for the colorimetric detection of cholera toxin. Anal. Chem., 79: 1356-1361. DOI: $10.1021 / \mathrm{ac} 061462 \mathrm{j}$

Shibata, H., M. Iimuro, N. Uchiya, T. Kawamori and M. Nagaoka et al., 2003. Preventive effects of Cladosiphon fucoidan against Helicobacter pylori infection in Mongolian gerbils. Helicobactor, 8: 59-65. DOI: 10.1046/j.1523-5378.2003.00124.x

Singaravelu, G., J.S. Acrokiamary, V.G. Kumar and K. Govindaraju, 2007. A novel extracellular synthesis of monodisperse gold nanpatricles using marine alga, Sargassum wightii Greville. Coll. Surf. B., 57: 97-101. DOI: 10.1016/j.colsurfb.2007.01.010

Sun, X.P., Z.L. Zhang, B.L. Zhang, X.D. Dong and S.J. Dong et al., 2003. Preparation of gold nanoparticles protected with polyelectrolyte. Chinese Chem. Lett., 14: 866-869. http://www.imm.ac.cn/journal/ccl/1408/140828866-02-611-p4.pdf

Teruya, T., T. Konishi, S. Uechi, H. Tamaki and M. Tako, 2007. Anti-proliferative activity of oversulfated fucoidan from commercially cultured Cladosiphon okamuranus TOKIDA in U937 cells. Inter. J. Biol. Macromol., 41: 221-226. DOI: 10.1016/j.ijbiomac.2007.02.010

Yang, J.W., S.Y. Yoon, S.J. Oh, S.K. Kim and K.W. Kang, 2006. Bifunctional effects of fucoidan on the expression of inducible nitric oxide synthase. Biochem. Biophys. Res. Commun., 346: 345-350. DOI: 10.1016/j.bbrc.2006.05.135

Zhao, W., W. Chiuman, J.C.F. Lam, S.A. McManus and W. Chen et al., 2008. DNA aptamer folding on gold nanoparticles: From colloid chemistry to biosensors. J. Am. Chem. Soc., 130: 3610-3618. DOI: $10.1021 / \mathrm{ja} 710241 \mathrm{~b}$ 